\title{
Differentiation of human stem cells is promoted by amphiphilic pluronic block copolymers
}

This article was published in the following Dove Press journal:

International Journal of Nanomedicine

7 September 2012

Number of times this article has been viewed

\author{
Ayșegül Doğan' \\ Mehmet E Yalvaç ${ }^{1,2}$ \\ Fikrettin Șahin' \\ Alexander V Kabanov ${ }^{3-5}$ \\ András Palotás ${ }^{6}$ \\ Albert A Rizvanov ${ }^{7}$ \\ 'Department of Genetics and \\ BioEngineering, College of Engineering \\ and Architecture, Yeditepe University, \\ Istanbul, Turkey; ${ }^{2}$ Center for Gene \\ Therapy, Nationwide Children's \\ Hospital, Ohio State University, \\ Columbus, OH, USA; ${ }^{3}$ Center for \\ Drug Delivery and Nanomedicine, \\ ${ }^{4}$ Department of Pharmaceutical \\ Sciences, College of Pharmacy, \\ Durham Research Center, University \\ of Nebraska Medical Center, Omaha, \\ NE, USA; ${ }^{5}$ Laboratory of Chemical \\ Design of Bio-nano-materials, \\ Department of Chemistry, Mikhail V \\ Lomonosov Moscow State University, \\ Moscow, Russia; ${ }^{6}$ Asklepios-Med, \\ Szeged, Hungary; ${ }^{7}$ Institute of \\ Fundamental Medicine and Biology, \\ Kazan (Volga Region) Federal \\ University, Kazan, Russia
}

Correspondence: Fikrettin Șahin Department of Genetics and BioEngineering, College of Engineering and Architecture, Yedpitepe University, 26 Ağustos Campus, Kayisdagi cad, Kayisdagi, TR-34755 Istanbul, Turkey Tel +902165780935

Email fsahin@yeditepe.edu.tr

András Palotás

Asklepios-Med, H-6722 Szeged,

Kossuth Lajos sgt 23, Hungary

Tel +36 302556225

Email palotas@asklepios-med.eu

\begin{abstract}
Stem cell usage provides novel avenues of tissue regeneration and therapeutics across disciplines. Apart from ethical considerations, the selection and amplification of donor stem cells remain a challenge. Various biopolymers with a wide range of properties have been used extensively to deliver biomolecules such as drugs, growth factors and nucleic acids, as well as to provide biomimetic surface for cellular adhesion. Using human tooth germ stem cells with high proliferation and transformation capacity, we have investigated a range of biopolymers to assess their potential for tissue engineering. Tolerability, toxicity, and their ability to direct differentiation were evaluated. The majority of pluronics, consisting of both hydrophilic and hydrophobic poly(ethylene oxide) chains, either exerted cytotoxicity or had no significant effect on human tooth germ stem cells; whereas F68 increased the multi-potency of stem cells, and efficiently transformed them into osteogenic, chondrogenic, and adipogenic tissues. The data suggest that differentiation and maturation of stem cells can be promoted by selecting the appropriate mechanical and chemical properties of polymers. It has been shown for the first time that F68, with its unique molecular characteristics, has a great potential to increase the differentiation of cells, which may lead to the development of new tissue engineering strategies in regenerative medicine.
\end{abstract}

Keywords: biopolymer, differentiation, human tooth germ stem cell (hTGSC), mesenchymal stem cell, pluronic, toxicity

\section{Introduction}

Various pathological conditions such as trauma, inflammation, degeneration, neoplastic proliferation, and surgical removal of tumors may lead to loss of skeletal tissue. Bony dehiscence can be treated by either replacement (ie, implanting grafts or prostheses) or by locally increasing osteogenesis to stimulate internal repair mechanisms of the body. ${ }^{1,2}$ The latter is addressed by regenerative medicine, including tissue engineering, whereby various sources are utilized to fabricate a desired biological structure. Stem cells have recently become prime candidates as they are capable of self-renewal and can differentiate into diverse specialized cellular types. These donor cells may be of embryonic or adult (post-natal) origin. However, induced pluripotent stem (iPS) cell sources have also become of interest whereby somatic cells are artificially transformed into pluripotency. Despite great enthusiasm and major progress, various ethical, legal, technical, safety, and other concerns still exist.

Complex regeneration of the skeletal system requires bony structures as well as cartilage. Multipotent mesenchymal stem cells (MSCs) can be converted into 
osteoblasts and chondrocytes, and can be found in virtually all postnatal organs, such as nonhematopoietic bone marrow, adipose tissue, synovial membrane, skeletal muscle, skin, trabecular bone, lung, and dental pulp. ${ }^{3}$ Recently, human tooth germ stem cells (hTGSCs), isolated from the third molars (wisdom teeth) of young adults, have been shown to exert MSC characteristics. The tooth germ (or tooth bud) is an aggregation of progenitor cells that forms a tooth, consisting of the dental papilla, the dental follicle, and the enamel organ. ${ }^{4}$ Tooth germs of third molars were reported to develop after the age of 6 years, but remain undifferentiated before this time. This may explain why MSCs derived from third molars are highly proliferative and multipotent. ${ }^{5}$ Moreover, stem cells residing in tooth germs have the ability to differentiate into several cell lines that originate from any of the three germ layers (ecto-, meso-, and endoderm). ${ }^{6}$

Instead of producing a mere cluster of newly formed cells, developing specific mechanical and structural properties for adequate functioning is essential in order to attain clinical relevance. To address this problem, stem cells can be cultured on natural or synthetic constructs that are intended to simulate a physiological microenvironment in order to promote tissue development. Such engineered extracellular matrices provide a foundation to adhere as well as grow, and are capable of supporting three-dimensional tissue formation. The properties of these biomimetic scaffolds can be manipulated to control cell behavior, including differentiation towards a specific lineage. ${ }^{7,8}$

Pluronics, also known as poloxamers, are biologically active polymers. Based on their relative biocompatibility and low cost, pluronics are good candidates in tissue engineering applications. ${ }^{9,10}$ Consisting of triblocks composed of hydrophilic poly(ethylene oxide) (PEO) and hydrophobic poly(propylene oxide) (PPO) chains in the form of $\mathrm{PEO}_{\mathrm{X}^{-}}$ $\mathrm{PPO}_{\mathrm{Y}}-\mathrm{PEO}_{\mathrm{X}}$, pluronics exhibit surfactant properties and can interact with biological membranes. For instance, pluronic unimers (single chain molecules) are able to incorporate into lipid bi-layers or even translocate through cell membranes. ${ }^{11,12}$ At high concentrations, pluronics aggregate into micelles between 10 and $100 \mathrm{~nm}$ in size, whereby PPO chains form the hydrophobic core enclosed by a hydrophilic PEO surface. As they can spontaneously incorporate ("solubilize") several molecules, such micelles may serve as vehicles for delivery of therapeutic agents, including drugs, growthfactors, or nucleic acids (genes). ${ }^{13}$ The various subtypes have specific structure-dependent properties. ${ }^{14}$ Pluronic F68 of the $\mathrm{PEO}_{76} \mathrm{PPO}_{29} \mathrm{PEO}_{76}$ formula, also known as poloxamer 188 , prevents cellular aggregation and protects cells from mechanical stress by attaching to the cellular membrane surface. ${ }^{15,16}$ Pluronic P85 ( $\left.\mathrm{PEO}_{26} \mathrm{PPO}_{40} \mathrm{PEO}_{26}\right)$ has the ability to inhibit various transporters, such as breast cancerresistant protein, multidrug resistance protein $2\left(\mathrm{MDR}_{2}\right)$, and P-glycoprotein (Pgp, also known as $\mathrm{MDR}_{1}$ ), thereby enhancing the cellular accumulation of anticancer and other drugs that are normally excluded from cells by these transporters. ${ }^{17}$ Pluronic F127 $\left(\mathrm{PEO}_{100} \mathrm{PPO}_{65} \mathrm{PEO}_{100}\right)$, or poloxamer 407, easily forms micelles and has been used as an efficient carrier for various hydrophobic drugs. It also interacts with cell surfaces, and can therefore be used as a scaffold system for carrying cells in various tissue engineering applications..$^{9,18,19}$

In this study, we hypothesize that these various polymeric biomaterials with distinctive chemical and mechanical properties could be employed to enhance the differentiation of hTGSCs to bony tissues as a potential system for skeletal repair. This study represents one of the first attempts to use synthetic amphiphilic block copolymers as biological response modulators in culturing and differentiating MSCs.

\section{Materials and methods Isolation and characterization of hTGSCs}

Human tooth germs were taken from the wisdom teeth of a 13-year-old patient following a routine dental treatment. Following approval from the Institutional Ethics Committee of Istanbul University (Turkey), written consent was obtained from the patient and his parents. hTGSCs were isolated and characterized as described previously by our group. ${ }^{20}$ Cells were removed from flasks with trypsinethylenediaminetraacetic acid (Invitrogen, Paisley, UK) and incubated with primary antibodies diluted in phosphatebuffered saline (PBS; cat no 10010, pH 7,4; Invitrogen) for 1 hour. Primary antibodies against CD29 (cat no BD556049), CD34 (cat no SC-51540), CD45 (cat no SC-70686), CD90 (cat no SC-53456), CD105 (cat no SC-71043), CD133 (cat no SC-65278), CD166 (cat no SC-53551) (Santa Cruz Biotechnology Inc, Santa Cruz, CA), and CD73 (cat no BD 550256) (Zymed, San Francisco, CA) were used in this study. Cells were washed with PBS to discard excess antibodies and incubated with fluorescein isothiocyanate-conjugated chicken anti-mouse secondary antibodies (cat. no. SC-2989, $200 \mu \mathrm{g} / 0.5 \mathrm{~mL}$; Santa Cruz Biotechnology) at $4^{\circ} \mathrm{C}$ for 1 hour, except for CD29, against which chromophore-containing phycoerythrin protein-conjugated monoclonal antibody was used. The flow cytometry analysis of cells was completed using a Becton Dickinson FACSCalibur (Becton Dickinson, San Jose, CA) flow cytometry system. 


\section{Preparation of pluronic solutions}

Solutions of pluronic block copolymers were prepared according to previously described protocols. ${ }^{21}$ Briefly, P85, F68, and F127 (Badische Anilin und Soda-Fabrik, Ludwigshafen-am-Rhein, Germany) were dissolved into PBS at a $10 \%(\mathrm{w} / \mathrm{v})$ concentration by vortexing and incubating on ice, and were subsequently diluted to $1 \%(\mathrm{w} / \mathrm{v})$ in Dulbecco's modified Eagle's medium (Invitrogen, Carlsbad, CA). Following sterilization using a $0.2 \mu \mathrm{m}$ filter (Sartorius AG, Göttingen, Germany), the solution was then used as a stock solution and kept at $4^{\circ} \mathrm{C}$ until further use.

\section{Cytotoxicity assay}

Four separate concentrations $(0.01 \%, 0.02 \%, 0.05 \%$, and $0.1 \% \mathrm{w} / \mathrm{v}$ ) of the three pluronics were prepared in Dulbecco's modified Eagle's medium using 10\% fetal bovine serum (Invitrogen), and 1\% of penicillin, streptomycin, and amphotericin (Biological Industries, Beit Haemek, Israel) from the $1 \%$ stock solution. hTGSCs at passage number 2 were seeded onto 96 -well plates (Corning Glasswork, Corning, NY) at a concentration of 5000 cells/well followed by the addition of pluronics. Cell viability was measured by the 3-(4,5-di-methyl-thiazol2-yl)-5-(3-carboxy-methoxy-phenyl)-2-(4-sulfo-phenyl)2H-tetrazolium (MTS)-assay (CellTiter96 AqueousOne Solution; Promega, Southampton, UK) according to the manufacturer's instructions. Briefly, following the incubation of cells with pluronics for 24,48 , and 72 hours, $10 \mu \mathrm{L}$ of MTS reagent in $100 \mu \mathrm{L}$ of growth medium was added to each well, and was subsequently incubated for 2 hours at $37^{\circ} \mathrm{C}$. Absorbance at $490 \mathrm{~nm}$ was detected using an ELISA plate reader (Biotek, Winooski, VT).

\section{Differentiation of MSCs}

hTGSCs were induced to differentiate into osteo-, chondro-, and adipogenic cells according to the protocol used previously by our group. ${ }^{20}$ For osteo- and adipogenic differentiation, hTGSCs at passage number 2 were counted and seeded onto 24-well plates (Corning Glasswork) at a concentration of 15,000 cells/well, and StemPro osteo- and adipogenic differentiation medium was added (Invitrogen). Cells were then incubated either with or without pluronics in a humidified incubator supplemented with $5 \% \mathrm{CO}_{2}$ at $37^{\circ} \mathrm{C}$ for $7-10$ days with the differentiation media being changed every other day. For chondrogenic differentiation, a micromass culture system was applied as previously described. ${ }^{22}$ Briefly, $12.5 \mu \mathrm{L}$ medium droplets containing 250,000 cells were placed in the center of each well of a 24-well plate, and were then incubated for 2 hours to provide cellular attachment Subsequently, $500 \mu \mathrm{L}$ of StemPro chondrogenic medium (Invitrogen) was added, and the medium was changed every other day.

\section{Immunocytochemistry analysis}

hTGSCs were fixed in $2 \%(\mathrm{w} / \mathrm{v})$ of paraformaldehyde for 30 minutes at $4{ }^{\circ} \mathrm{C}$, and were subsequently washed three times for 5 minutes in PBS by gentle shaking. Permeabilization of cells was obtained by incubating them with $0.1 \%(\mathrm{v} / \mathrm{v})$ Triton-X 100 diluted in PBS for 5 minutes at room temperature. After rinsing in PBS three times for 5 minutes, samples were then incubated with $2 \%$ of goat serum (Sigma-Aldrich, Steinheim, Germany) diluted in PBS for 20 minutes at $4^{\circ} \mathrm{C}$ in order to block nonspecific binding of antibodies. Cells were then incubated with the following primary antibodies (Santa Cruz Biotechnology) overnight at $4^{\circ} \mathrm{C}$ : for osteogenic differentiation anti-collagen-type-1 (Santa Cruz \#59772) and antiosteocalcin (Santa Cruz \# 30044), for chondrogenic differentiation anti-collagen-type-2 (Santa Cruz \#28887), and for adipogenic differentiation anti-fatty-acid-binding-protein-4 (anti-FABP ${ }_{4}$ : Santa Cruz \#136150) primary antibodies were used. After labeling, cells were washed three times with PBS for 5 minutes to remove unbound primary antibodies, and were subsequently incubated with AleaFluor-488 goat anti-rabbit or goat antimouse immunoglobulin $\mathrm{G}$ (Invitrogen) secondary antibodies for 1 hour at $4^{\circ} \mathrm{C}$. Samples were subsequently rinsed three times with PBS for $5 \mathrm{~min}$, and cell nuclei were stained for 20 minutes at $4^{\circ} \mathrm{C}$ with diluted (1:1000) 4',6-di-amidino-2phenyl-indole (DAPI: Applichem, Darmstadt, Germany). After rinsing three times with PBS, cells were then observed under a fluorescence microscope (Nikon Eclipse TE200: Nikon, Tokyo, Japan).

\section{Real-time (RT) polymerase chain-reaction (PCR) analysis}

Primers for collagen type- $3, \mathrm{FABP}_{4}$, and cartilage-specific proteoglycan core protein (aggrecan) genes were designed by using Primer-BLAST online software from the National Center for Biotechnology (Bethesda, MD) and synthesized by Invitrogen. Other primer sequences were used as described elsewhere, ${ }^{23,24}$ and Table 1 delineates primers that were used in this study. Total RNAs from differentiated samples were isolated using High Pure RNA-isolation kit (Roche, Indianapolis, IN) according to the manufacturer's suggestions. cDNA was synthesized by using High Fidelity cDNA-synthesis kit (Roche). Reverse transcription polymerase chain reaction 
Table I Sense and anti-sense primers used in the study

\begin{tabular}{|c|c|c|c|}
\hline Primer & Sense $\left(5^{\prime}-3^{\prime}\right)$ & Anti-sense $\left(5^{\prime}-3^{\prime}\right)$ & Base-pair \\
\hline GAPDH & TGGTATCGTGGAAGGACTCA & GCAGGGATGATGTTCTGGA & 123 \\
\hline Osteonectin & ATGAGGGCCTGGATCTTCTT & CTGCTTCTCAGTCAGAAGGT & 576 \\
\hline Collagen II & GTGTGGAAGCCGGAGCCCTG & GGTCCTGGTTGCCCACTGGC & 170 \\
\hline Aggrecan & ACTGCTGCAGACCAGGAGGT & TCCTCGGGGGTGACGATGCT & 152 \\
\hline $\mathrm{FABP}_{4}$ & GGGTCACAGCACCCTCCTGA & TGGTGGCAAAGCCCACTCCTAC & 162 \\
\hline
\end{tabular}

Note: GAPDH, osteonectin, collagen 2, aggrecan, and $\mathrm{FABP}_{4}$ primers used in the study.

Abbreviations: GAPDH, glyceraldehyde-3-phosphate dehydrogenase; $\mathrm{FABP}_{4}$, fatty-acid-binding-protein 4.

(RT-PCR) using SYBR Green staining method was utilized to quantify mRNA levels of the genes. cDNAs were mixed with primers and SYBR-Premix ExTaq (TaKaRa, Shiga, Japan) in a final volume of $20 \mu \mathrm{L}$. The glycolysis-catalyzing housekeeping gene (GAPDH: glycer-aldehyde-3-phosphatede-hydrogenase) was used for normalization of data. All RTPCR experiments were done using iCycler RT-PCR system (Bio-Rad, Hercules, CA).

\section{Alkaline-phosphatase (ALP) activity}

ALP enzyme activity assay was performed to confirm osteogenic differentiation. Cells were trypsinized and centrifuged at $1000 \mathrm{rpm}$ for 5 minutes. Pellets were re-suspended in $500 \mu \mathrm{L}$ cell-lysis buffer containing $0.2 \%(\mathrm{v} / \mathrm{v})$ triton-X 100 in PBS, and were shaken at $850 \mathrm{rpm}$ for 30 minutes at room temperature. Subsequently, $25 \mu \mathrm{L}$ of protein lysate was mixed with $75 \mu \mathrm{L}$ of ALP ligand (Randox ALP detection kit; Randox, Antrim, UK) in a 96-well plate and incubated for 15 minutes followed by measuring the absorbance at $405 \mathrm{~nm}$ using an ELISA plate reader (Biotek) to detect enzyme activity.

\section{von Kossa staining}

von Kossa staining was performed to visualize calcium depositions, which are characteristic of osteogenic differentiation. Following 10 days of incubation with osteogenic medium, cells in a 24 -well plate were fixed with $2 \%(\mathrm{w} / \mathrm{v})$ of paraformaldehyde (Sigma-Chemical Co, St Louis, MO) at $4^{\circ} \mathrm{C}$ for $30 \mathrm{~min}$, rinsed with distilled water, and stained with vonKossa kit (BioOptica, Milano, Italy) according to the manufacturer's recommendations. Calcium depositions were identified using phase contrast light microscopy.

\section{Alcian blue staining}

Alcian blue stain sulfated proteoglycan deposits are indicative of functional chondrocytes. The staining solution was prepared by dissolving $1.00 \mathrm{~g}$ of Alcian blue dye (SigmaChemical Co) into $100 \mathrm{~mL}$ of $3 \%$ (v/v) acetic acid. Cells were fixed with $2 \%(\mathrm{w} / \mathrm{v})$ paraformaldehyde for 30 minutes and stained with Alcian-blue staining solution for 30 minutes. Following incubation, cells were washed three times with PBS, and samples were observed using phase contrast light microscopy. ${ }^{24}$

\section{Oil-red staining}

Oil-red was used to visualize lipid vesicles, which are characteristic of adipogenic differentiation. A stock solution was prepared by dissolving $0.5 \mathrm{~g}$ oil-red (Sigma-Chemical Co) into $100 \mathrm{~mL}$ of isopropanol. Cells were fixed with $2 \%(\mathrm{w} / \mathrm{v})$ of paraformaldehyde for 30 minutes, rinsed with PBS, and stained with oil-red in PBS (diluted 6:4) for 1 hour. The preparation was then washed with PBS and assessed under phase contrast light microscopy.

\section{Identification of fatty acids}

Fatty acids were assessed to evaluate the effect of pluronics on the membrane fatty acid profile. Isolation was performed according to a previously described protocol ${ }^{25}$ modified by our group. In short, hTGSCs were counted and seeded in Petri-dishes at a concentration of $1 \times 10^{6}$ cells/ dish in growth media supplemented with $0.05 \%(\mathrm{w} / \mathrm{v})$ of F68, P85, or F127. Cells were trypsinized on the third day and pellets were collected after 5 minutes centrifugation at $1000 \mathrm{rpm}$. Following the transfer to glass screw cap tubes, $1 \mathrm{~mL}$ of $1.2 \mathrm{M} \mathrm{NaOH}$ dissolved in $50 \%$ aqueous methanol was added to the pellets and incubated in a water bath at $100^{\circ} \mathrm{C}$ for 30 minutes. After cooling at room temperature for 25 minutes, $2 \mathrm{~mL}$ of $54 \%$ (w/v) $6 \mathrm{~N} \mathrm{HCl}$ in $46 \%$ aqueous methanol (v/v) was added and incubated in a water bath at $80^{\circ} \mathrm{C}$ for 10 minutes. Samples were than cooled on ice, and $1.25 \mathrm{~mL}$ of hexane solution containing $50 \%$ methyl tert-butyl ether (MTBE) was administered to form a top and bottom phase in the tubes. The bottom phase was removed with a Pasteur pipette, and the top phase was mixed with $3 \mathrm{~mL}$ of $0.3 \mathrm{M} \mathrm{NaOH}$, cleaned with anhydrous sodium sulfate, and transferred into a $1 \mathrm{~mL}$ clean glass vial for analysis using a midi-fatty acid analysis system (Agilent Technologies, Wilmington, DE). 

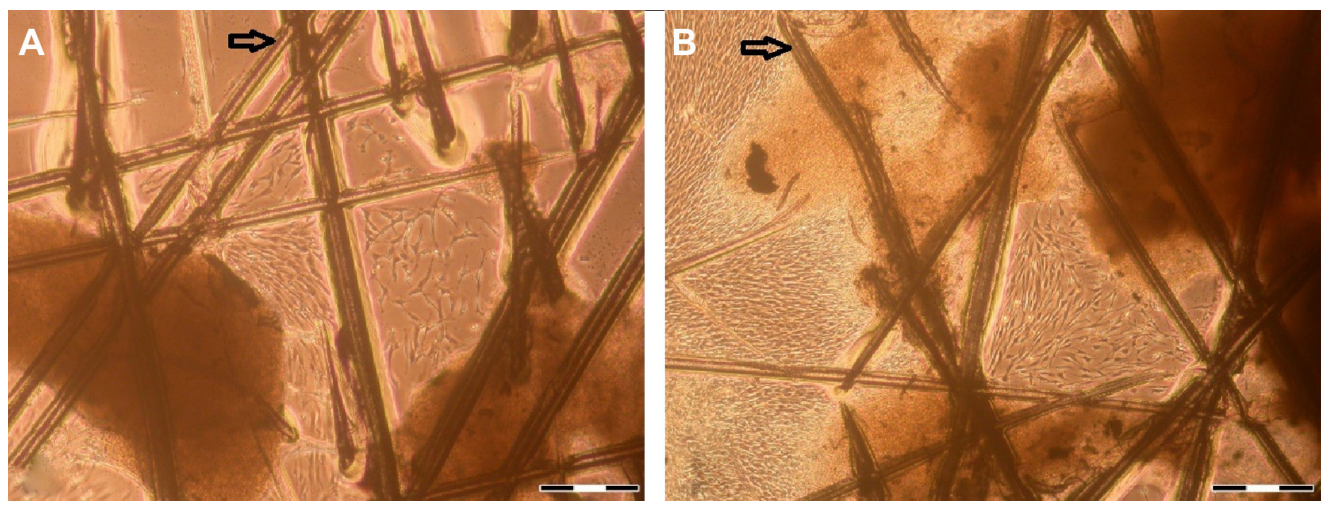

Figure I Isolated hTGSCs. hTGSCs at (A) day 3 and (B) day 8.

Notes: Scale bar $=400 \mu \mathrm{m}$. Cells started to grow from human tooth germ tissues that were dismembered and minced by scalpel (resulting scratches on the flask surface are marked by arrows).

Abbreviations: hTGSCs, human tooth germ stem cells.
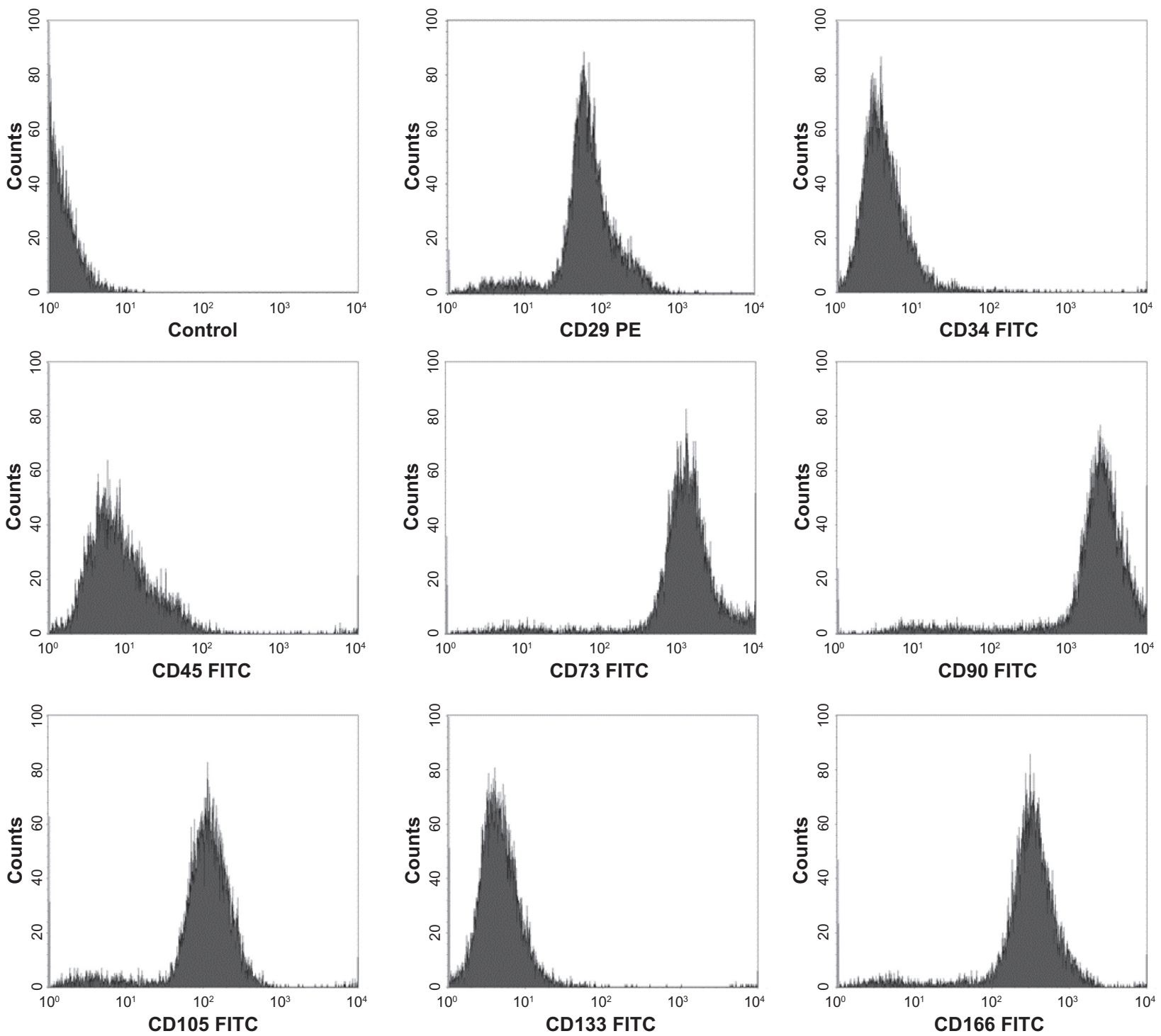

Figure 2 Immunophenotypic characteristics of hTGSCs.

Notes: Flow cytometry analysis showing hTGSCs express MSC-associated cell surface antigens (CD29, CD73, CD90, CDI05 and CDI66), but not markers characteristic of hematopoietic lines (CD34, CD45 and CDI33).

Abbreviations: hTGSCs, human tooth growth cells; MSC, mesenchymal stem cells. 


\section{Statistical calculations}

Standard errors and $t$-test values were calculated using GraphPad Prism 5 (GraphPad, La Jolla, CA) software. Differences were considered to be significantly significant at $P$ values of less than 0.05 .

\section{Results}

\section{Isolation and characterization of hTGSCs}

hTGSCs were successfully isolated in culture dishes from tooth explants (Figure 1), and were characterized for their surface markers by flow cytometry. Cells were shown to be positive for MSCs markers (CD29, CD73, CD90, CD105, CD166) and proved to be negative for hematopoietic markers (CD34, CD45, CD133) (Figure 2).

\section{Cytotoxicity of pluronics in hTGSCs}

The impact of various pluronics on hTGSC viability was tested at different concentrations $(0.01 \%, 0.02 \%, 0.05 \%$, and $0.1 \% \mathrm{w} / \mathrm{v}$ ) and exposure times $(24,48$, and 72 hours). No cytotoxic effect of these copolymers was detected on resting samples (data not shown). F68 and F127 have proved to be safe during transformation into osteo-, chondro-, and adipogenic directions; however, P85 caused cell death in differentiating hTGSCs. Showing toxic effects in a dosedependent manner, P85 at $0.05 \%$ appeared to be lethal on the first day of differentiation, whereas the lowest dose of $0.01 \%$ demonstrated toxic effects on day 55 only (Figure 3 ). Since P85 appeared to be noxious, it was excluded from further studies involving differentiation.

\section{Immunocytochemistry}

In order to confirm the differentiation of cells, hTGSCs were labeled with specific antibodies against markers of osteogenic (collagen type-1, osteocalcin), chondrogenic (collagen type-2), and adipogenic $\left(\mathrm{FABP}_{4}\right)$ transformation. The results indicate that all samples, including those treated with F68 and F127, were positive for these markers (Figure 4).

\section{RT-PCR analysis}

Treatment of hTGSCs with F68 resulted in a significant increase in mRNA levels of osteonectin, collagen type-2, aggrecan, and $\mathrm{FABP}_{4}$ (Figure 5). In contrast, F127 had either no effect (collagen type-2) or considerably suppressed (osteonectin, aggrecan, $\mathrm{FABP}_{4}$ ) mRNA expression.

\section{ALP activity in differentiating hTGSCs}

ALP is an osteogenic marker found at high concentrations in the growing bone and teeth where it plays essential roles
A

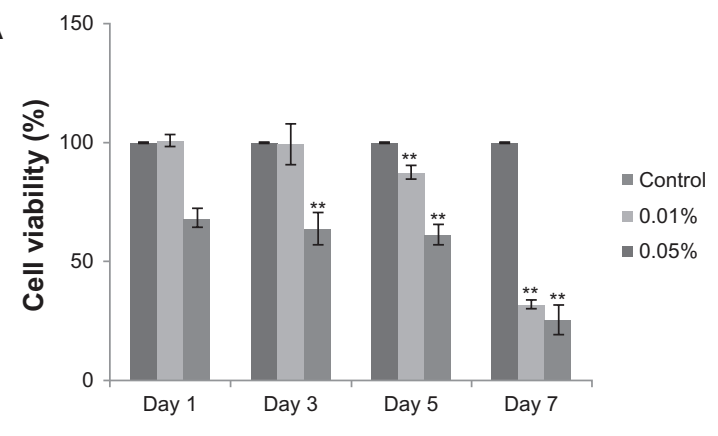

B
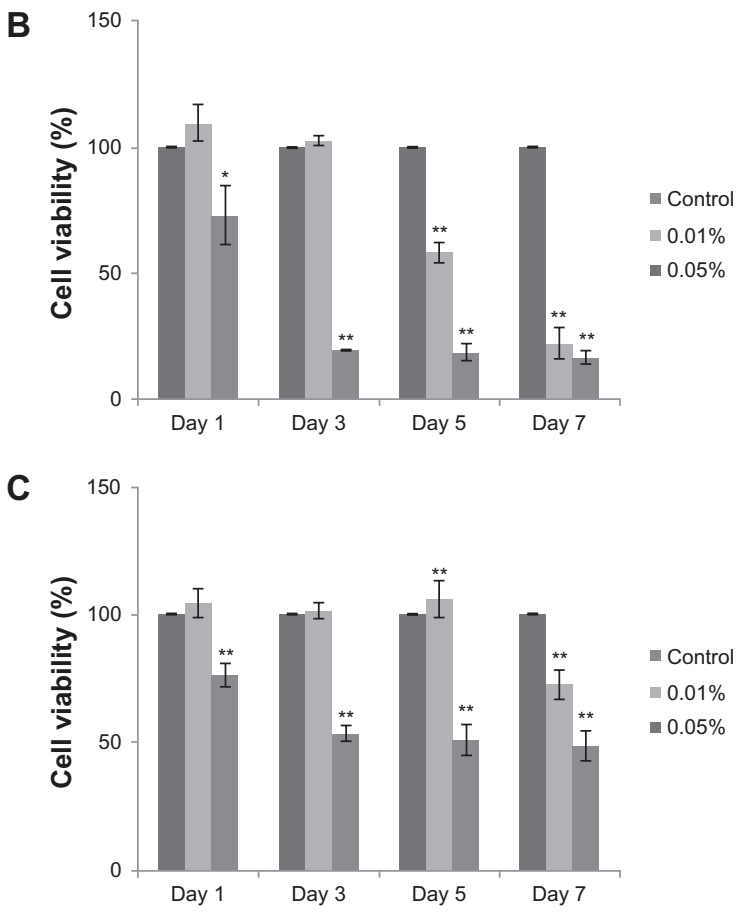

Figure 3 Cytotoxicity of P85. Pluronic P85 exerted toxic effects on day I at $0.05 \%$ concentration, and from day 5 at $0.01 \%$ strength during (A) osteo-, (B) chondro-, and (C) adipogenic differentiation.

Notes: hTGSCs without P85 treatment were used as controls. ${ }^{*} P<0.05$, **P $<0.001$. Abbreviations: hTGSCs, human tooth germ growth cells; $P$, probability level.

for the deposition of minerals. ${ }^{26}$ F68 and F127 significantly increased ALP levels during the osteogenic differentiation of cells (Figure 6).

\section{von Kossa staining}

Controls and F68-treated samples exhibited considerable calcium deposition that is characteristic of osteogenic transformation, and F127 cells appeared to have less mineralization (Figure 7).

\section{Alcian blue staining}

All samples stained positive with Alcian blue, indicating mucopolysaccharide and glycosaminoglycan formation during the course of chondrogenic differentiation (Figure 8). 

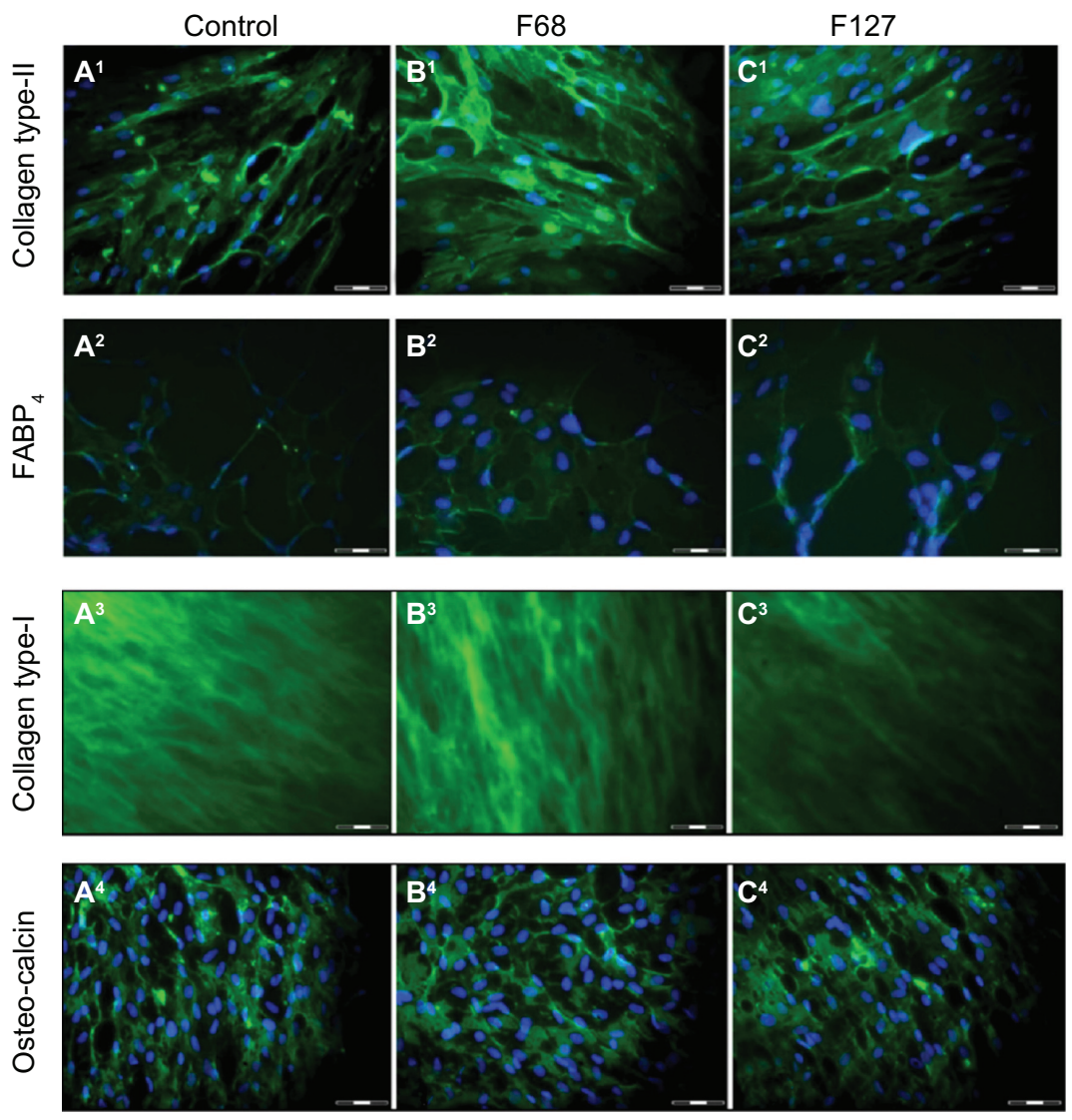

Figure 4 Immunocytochemical analysis of hTGSCs treated with various pluronics. Specific antibodies for osteogenic (collagen type-I, osteocalcin), chondrogenic (collagen type-2), and adipogenic $\left(\mathrm{FABP}_{4}\right)$ markers were used. Cells in $\left(\mathbf{A}^{\prime}-\mathbf{A}^{4}\right)$ control, $\left(\mathbf{B}^{\prime}-\mathbf{B}^{4}\right)$ F68-treated, and $\left(\mathbf{C}^{\prime}-\mathbf{C}^{4}\right)$ FI27-treated groups were all labeled positively, confirming successful differentiation.

Note: Scale bar $=100 \mu \mathrm{m}$.

Abbreviations: hTGSCs, human tooth germ growth cells; $\mathrm{FABP}_{4}$, fatty-acid-binding-protein 4.
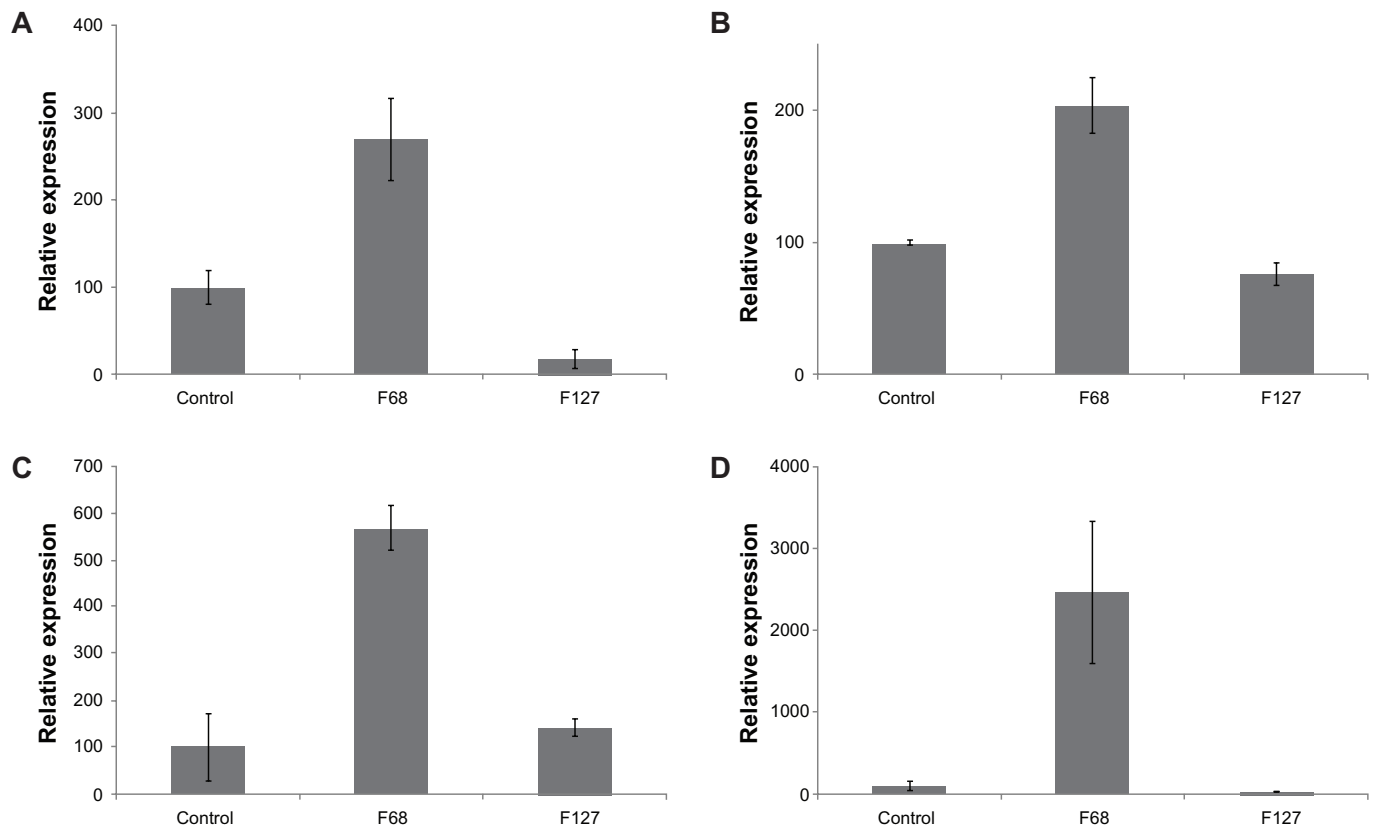

Figure 5 Comparison of mRNA expression levels of differentiation marker genes. F68 increased mRNA levels of $(\mathbf{A}) \mathrm{FABP}_{4}$, (B) osteonectin, $(\mathbf{C})$ collagen type-2, and (D) aggrecan.

Abbreviation: mRNA, messenger ribonucleic acid. 


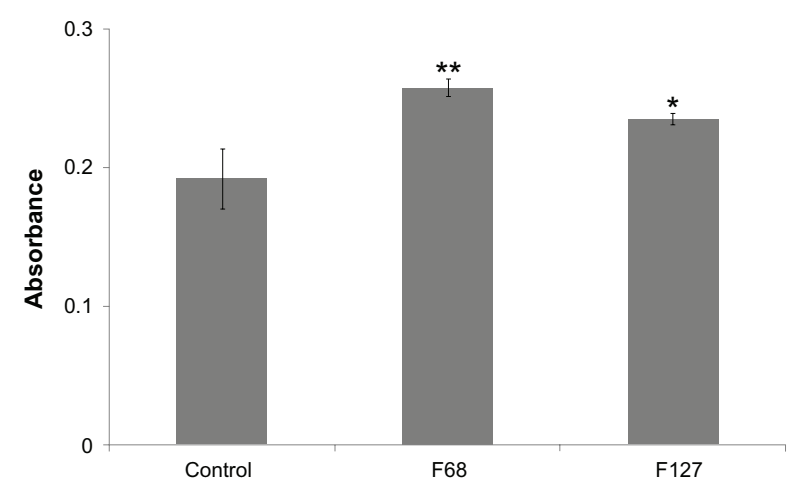

Figure 6 Analysis of ALP activity.

Notes: ALP was detected to be highest in F68 group during osteogenic differentiation. $* P<0.05$, $* * P<0.001$.

Abbreviations: ALP, alkaline-phosphatase; $P$, probability level.

\section{Oil-red staining}

Upon adipogenic differentiation, neutral triglycerides and lipid vesicles were stained with oil-red in all experimental groups (Figure 9).

\section{Effect of pluronics on fatty acid profile of hTGSCs}

F68 has profoundly altered the lipid composition of hTGSCs. Moreover, it caused the depletion of a few saturated fatty acids, in particular 16:0 fatty, 20:04 w6c, and led to the incorporation of four new unsaturated ones (16:1w9c, $16: 1 \mathrm{w} 7 \mathrm{c}, 18: 2 \mathrm{w} 6 \mathrm{c}$, and $22: 1 \mathrm{w} 9 \mathrm{c}$ alcohol) into the cellular membrane (Figure 10). In contrast, hTGSCs replaced one of their characteristic lipid groups (called summed feature 4) to another fatty acid (18:2w6c) because of P85, whereas F127 caused an addition of 22:1w9c alcohol into the cellular lipid bi-layer.

\section{Discussion}

The capacity of the human body to regenerate itself greatly varies depending on the affected tissue. For instance, epithelial cells can repair skin wounds throughout life; whereas connective tissues including bone or cartilage have limited repair ability, and terminally differentiated structures such as the heart or brain cannot compensate for the large-scale loss of cells seen after myocardial infarction or degenerative neurological conditions. Transplantation is a viable treatment method for select end-stage organ failures (eg, heart, kidney, liver), but is not applicable in early stages. Furthermore, transplantation involves immunogenic responses, and is restricted by the limited number of donors. Function permitting, such as with the skeletal system, an alternative option is replacement with a bio-prosthesis; these artificial materials, however, are associated with several foreign body related complications such as bone resorption that may lead to the loosening of the implant or even fractures. Cell-based therapies have therefore emerged as new potential therapeutic options in structures with low regenerative potential. The focus has shifted to stem cells because of their unique proliferation and differentiation capacity. Due to the ethical problems over-shadowing the clinical application of embryonic stem cells, post-natal sources have become of much interest. However, the number of pluripotent stem cells is limited in the adult body, although there are a few sites such as bone marrow, adipose tissue, and dental material that can serve as potential well-springs. ${ }^{27}$ Bone marrow derived MSCs can be easily isolated and expanded in vitro, but several factors such as painful surgical isolation procedures, contamination risk, bone-related diseases and low yield of isolated cells limit their usage. ${ }^{28}$ As an alternative source, valuable by-products of routine dental operations that cause no ethical or safety controversies are hTGSCs. In addition to efficient isolation, they have MSC properties and were shown to differentiate into many cell types including osteo-, adipo-, and chondrocytes, as well as neurons..$^{20,29}$ Moreover, surgery-related issues are not independent risks or concerns when isolating hTGSCs, as they are harvested from dental waste material after intervention due to other (ie, dental) reasons.
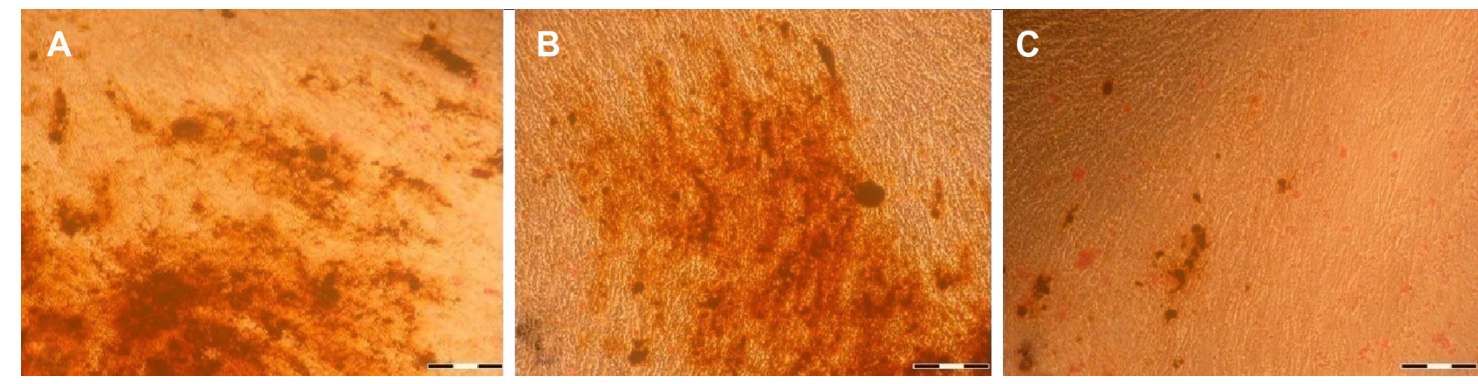

Figure 7 vonKossa staining. Calcium depositions in (A) control, (B) F68, and (C) Fl27-treated groups were detected during osteogenic differentiation. Note: Scale bar $=400 \mu \mathrm{m}$. 

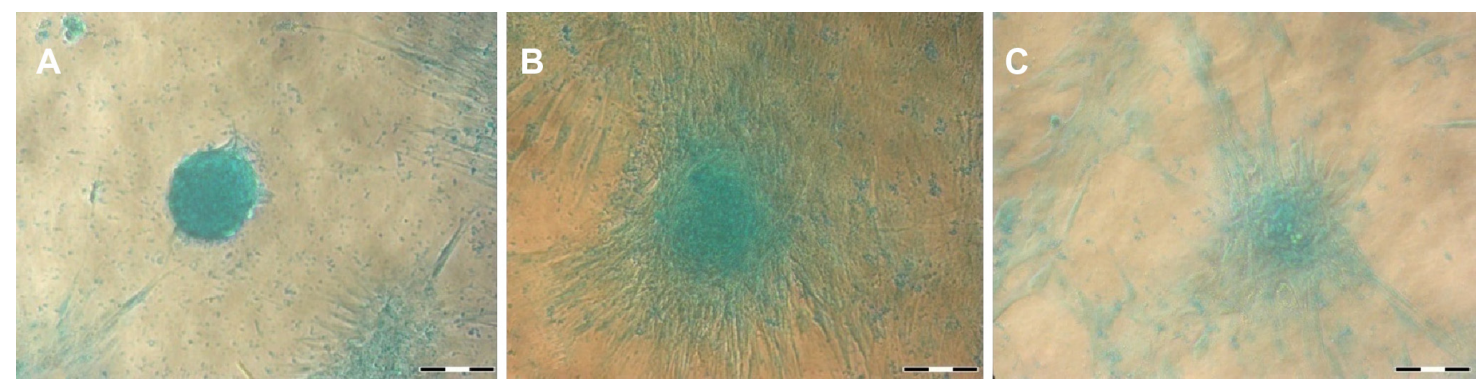

Figure 8 Alcian-blue staining. (A) control, (B) F68, and (C) FI27-treated groups all stained positive with Alcian-blue, confirming chondrogenic differentiation. Note: Scale bar $=400 \mu \mathrm{m}$.

In addition to stem cells, novel tissue engineering approaches utilize suitable biomaterials that provide a molecular matrix as a biomimetic surface for cell attachment to promote proliferation and differentiation. These scaffolds must be nontoxic and biocompatible in order to develop cell-seeded bio-engineered tissues. Various materials (natural and synthetic, biodegradable and permanent) have been investigated. F68, P85, and F127 are widely used, commercially available block copolymers. This study, therefore, tested the effects of these pluronics on the differentiation of hTGSCs derived from human impacted third molars.

Here we showed that the differentiation efficiency of hTGSCs was increased by F68, and cells in the presence of this pluronic showed the highest expression of osteo-, chondro-, and adipogenic-related genes when compared to baseline or treatment with other copolymers. Indeed, F68 is known to promote cellular attachment to the culture dish by interacting with lipid bi-layers, which eventually stimulates cell growth in vitro. ${ }^{30,31} \mathrm{~F} 68$ also raised the unsaturated fatty acid levels of cell membranes in this study. This is in line with the fact that F68 can incorporate into bio-membranes and modify their structural and functional properties by altering the lipid composition. ${ }^{32}$ An elevated unsaturated ratio is known to increase membrane fluidity, ${ }^{33}$ which may generally promote cellular differentiation.

Transforming hTGCSs into the osteogenic direction has obvious clinical relevance as it may serve as a springboard for managing skeletal dehiscence via tissue engineering modes. Complex reconstruction of bony structures, however, requires cartilage as well, which has limited blood supply and their cells are terminally differentiated into chondrocytes that are unable to proliferate and regenerate effectively. ${ }^{34}$ F68 demonstrated an ability to promote the differentiation of hTGSCs into both osteo- and chondrogenic lines, therefore it might be a good candidate in regenerative medicine for skeletal lesions. In addition, the use of adipogenic progenitor cells derived from hTGSCs may also emerge as a promising approach for cell-based adipose tissue engineering with various clinical implications: fatty defects are usually seen after traumas, deep burns, tumor resections such as mastectomies, and are important in cosmetic facial problems involving the cheek, chin, and jaw. ${ }^{35-37}$

In contrast, P85 had no significant impact on the fatty acid profile of hTGSCs; however, it displayed considerable cytotoxic effect during differentiation even at low concentrations $(0.01 \%, 0.05 \%)$. P85 is generally used for the inhibition of the Pgp drug efflux system, which is crucial for improved
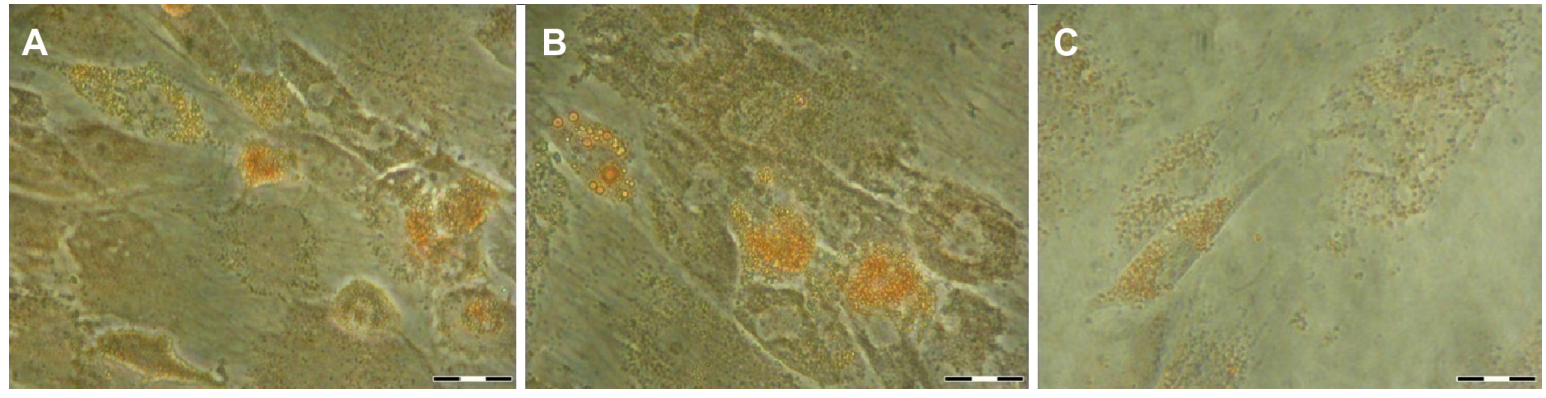

Figure 9 Oil-red staining. (A) control, (B) F68, and (C) FI27-treated groups all stained positive with oil-red, confirming the presence of neutral triglycerides and lipid vesicles that are characteristic of adipogenic differentiation.

Note: Scale bar $=400 \mu \mathrm{m}$. 

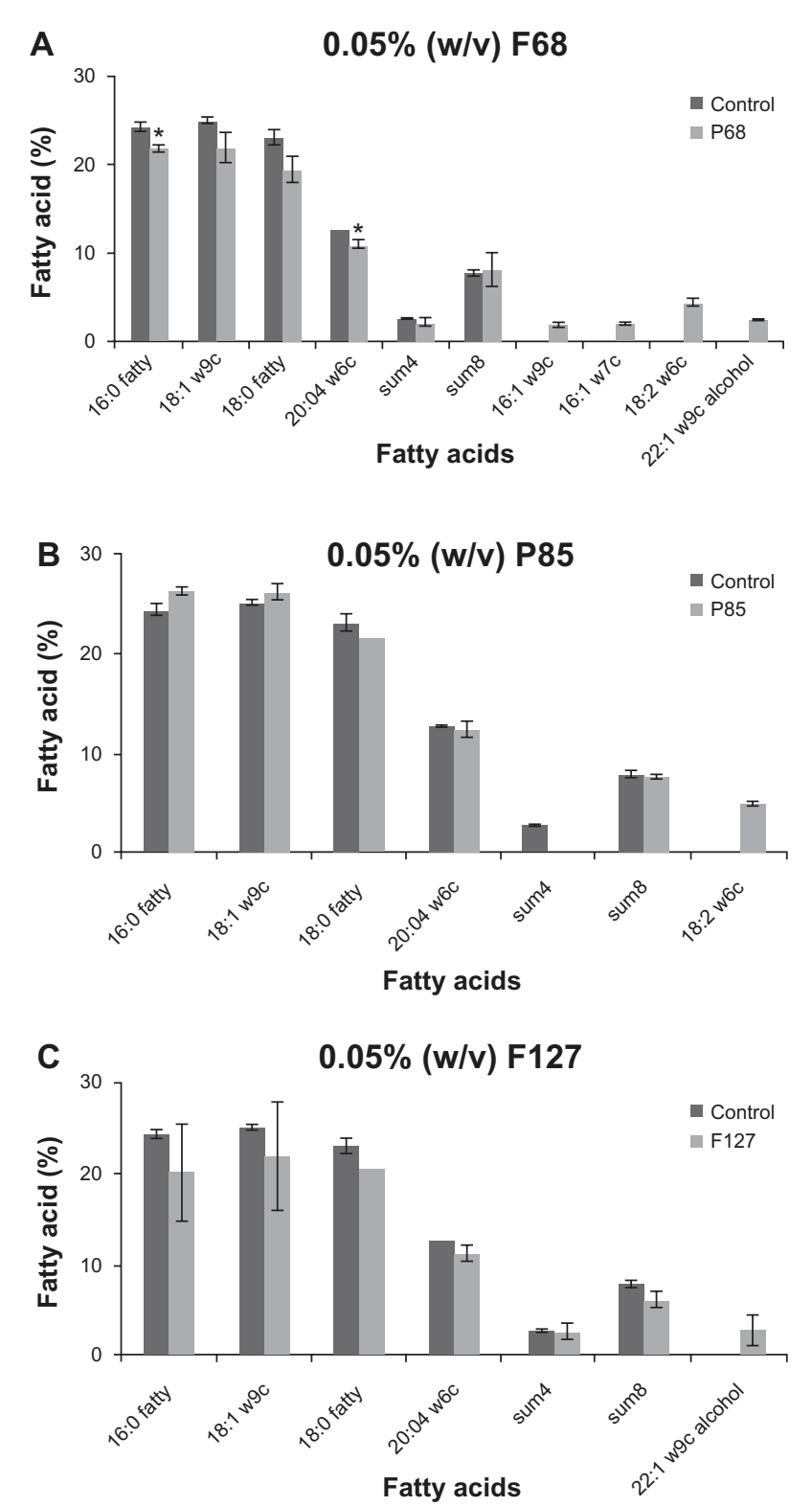

Figure 10 Fatty acid profile of cells. (A) treatment with F68 caused a significant decrease in the levels of certain saturated lipids (16:0 fatty, 20:04 w6c), and triggered cells to produce new unsaturated fatty acids (16:Iw9c (I.9\%), I6:Iw7c (2.2\%), 18:2w6c (4.6\%), and 22: I w9c alcohol). (B) F85 group showed altered sum4 (summed featured 4) and I8:I $\omega 9 \mathrm{t}$ levels, whereas (C) administration of $\mathrm{FI} 27$ resulted in the introduction of 22: I w9c alcohol into the lipid bi-layer.

Note: $* P<0.05$.

Abbreviation: $P$, probability level.

delivery of Pgp-dependent molecules through the blood-brain barrier, intestinal tight-junctions, and MDR-tumors. ${ }^{38,39}$ As such, P85 may facilitate the intake of various chemicals such as dexamethasone that are used in differentiation experiments, causing their accumulation in high concentrations which leads to cell death. However, P85 appeared to be toxic only during differentiation. hTGSCs might undergo major changes in cell metabolism, including over-expressing drug efflux transporters during proliferation. Inhibition of Pgp-dependent mechanisms during sensitive cell growth by this pluronic could explain the phase-specific toxicity of P85.

F127 has many favorable noxious properties and is highly water-soluble at low temperatures..$^{40,41}$ Being prone to micelleformation, F127-mediated controlled release of growth factors was shown to increase differentiation efficiency of MSCs. ${ }^{910,42}$ This study, unlike the previous reports, used F127 as an ingredient in the differentiation medium at a very low concentration $(0.05 \%)$. The results indicate that although ALP activity is increased, this biopolymer has very little effect on the expression of differentiation-related genes. As such, F127 perhaps has more relevance in tissue engineering when used as a micelle and combined with various growth factors.

This is the first study to demonstrate the impact of these pluronics on membrane fatty acid profiles along with the expression of differentiation markers in a relatively new stem cell source (hTGSCs). Of all poloxamers assessed, F68 has proven to be a promising synthetic polymeric agent that might lead to the development of highly productive regenerative therapies for various bone, cartilage, and adipose tissue defects. These data also indicate that hTGSC-derived differentiation and maturation can be promoted by tuning the mechanical and chemical properties of the polymer scaffold by applying the most appropriate PEO/PPO chain blocks. This feature extends the potential applications of this postnatal progenitor cell-based technology for transplantable large tissue-engineered constructs.

\section{Acknowledgments}

The authors would like to express their gratitude to Dr Burcin Keskin for her assistance in flow cytometry analysis. This study was supported by Yeditepe University (Turkey), grants from the Russian Foundation for Basic Research, Pharmaceutical Research and Education Center of Kazan (Volga Region) Federal University (Russia), and by Asklepios-Med (Hungary). AVK acknowledges support in part from the United States National Institutes of Health grants 2RO1 CA89225, 1R01 CA116591, and from the Russian Ministry of Science and Education (contract 11.G34.31.0004 and 16.552.11.7008).

\section{Disclosure}

The authors report no conflicts of interest in this work.

\section{References}

1. Perry CR. Bone repair techniques, bone graft, and bone graft substitutes. Clin Orthop Relat Res. 1999;360:71-86.

2. Heary RF, Schlenk RP, Sacchieri TA, Barone D, Brotea C. Persistent iliac crest donor site pain: independent outcome assessment. Neurosurgery. 2002;50(3):510-516. 
3. da Silva Meirelles L, Chagastelles PC, Nardi NB. Mesenchymal stem cells reside in virtually all post-natal organs and tissues. J Cell Sci. 2006;119(Pt 11):2204-2213.

4. Liu H, Li W, Shi S, Habelitz S, Gao C, Denbesten P. MEPE is downregulated as dental pulp stem cells differentiate. Arch Oral Biol. 2005;50(11):923-928.

5. Kerkis I, Kerkis A, Dozortsev D, et al. Isolation and characterization of a population of immature dental pulp stem cells expressing OCT-4 and other embryonic stem cell markers. Cells Tissues Organs. 2006;184(3-4):105-116.

6. Ikeda E, Yagi K, Kojima M, et al. Multipotent cells from the human third molar: feasibility of cell-based therapy for liver disease. Differentiation. 2008;76(5):495-505.

7. Kellar RS, Landeen LK, Shepherd BR, Naughton GK, Ratcliffe A, Williams SK. Scaffold-based three-dimensional human fibroblast culture provides a structural matrix that supports angiogenesis in infarcted heart tissue. Circulation. 2001;104(17):2063-2068.

8. Kwan MD, Slater BJ, Wan DC, Longaker MT. Cell-based therapies for skeletal regenerative medicine. Hum Mol Genet. 2008;17(R1) R93-R98.

9. Vashi AV, Keramidaris E, Abberton KM, et al. Adipose differentiation of bone marrow-derived mesenchymal stem cells using Pluronic F-127 hydrogel in vitro. Biomaterials. 2008;29(5):573-579.

10. Huang JW, Chen WJ, Liao SK, Yang CY, Lin SS, Wu CC. Osteoblastic differentiation of rabbit mesenchymal stem cells loaded in A carrier system of Pluronic F127 and Interpore. Chang Gung Med J. 2006;29(4):363-372.

11. Sahay G, Batrakova EV, Kabanov AV. Different internalization pathways of polymeric micelles and unimers and their effects on vesicular transport. Bioconjug Chem. 2008;19(10):2023-2029.

12. Sahay G, Gautam V, Luxenhofer R, Kabanov AV. The utilization of pathogen-like cellular trafficking by single chain block copolymer. Biomaterials. 2010;31(7):1757-1764.

13. Kabanov AV, Batrakova EV, Sriadibhatla S, Yang Z, Kelly DL, Alakov VY. Polymer genomics: shifting the gene and drug delivery paradigms. J Control Release. 2005;101(1-3):259-271.

14. Kabanov AV, Batrakova EV, Alakhov VY. Pluronic block copolymers as novel polymer therapeutics for drug and gene delivery. $J$ Control Release. 2002;82(2-3):189-212.

15. Murhammer DW, Goochee CF. Sparged animal cell bioreactors: mechanism of cell damage and Pluronic F-68 protection. Biotechnol Prog. 1990;6(5):391-397.

16. Jordan M, Eppenberger HM, Sucker H, Widmer F, Einsele A. Interactions between animal cells and gas bubbles: the influence of serum and pluronic F68 on the physical properties of the bubble surface. Biotechnol Bioeng. 1994;43(6):446-454.

17. Witt KA, Huber JD, Egleton RD, Davis TP. Pluronic p85 block copolymer enhances opioid peptide analgesia. J Pharmacol Exp Ther. 2002;303(2):760-767.

18. Erukova VY, Krylova OO, Antonenko YN, Melik-Nubarov NS Effect of ethylene oxide and propylene oxide block copolymers on the permeability of bilayer lipid membranes to small solutes including doxorubicin. Biochim Biophys Acta. 2000;1468(1-2): 73-86.

19. Cunha C, Panseri S, Villa O, Silva D, Gelain F. 3D culture of adult mouse neural stem cells within functionalized self-assembling peptide scaffolds. Int J Nanomedicine. 2011;6:943-955.

20. Yalvac ME, Ramazanoglu M, Gumru OZ, Sahin F, Palotás A, Rizvanov AA Comparison and optimisation of transfection of human dental follicle cells, a novel source of stem cells, with different chemical methods and electroporation. Neurochem Res. 2009;34(7):1272-1277.

21. Exner AA, Krupka TM, Scherrer K, Teets JM. Enhancement of carboplatin toxicity by Pluronic block copolymers. J Control Release. 2005;106(1-2):188-197.

22. Zhang Z, al-Rubeai M, Thomas CR. Effect of Pluronic F-68 on the mechanical properties of mammalian cells. Enzyme Microb Technol. 1992;14(12):980-983.
23. Alliot-Licht B, Bluteau G, Magne D, et al. Dexamethasone stimulates differentiation of odontoblast-like cells in human dental pulp cultures. Cell Tissue Res. 2005;321(3):391-400.

24. Lisignoli G, Cristino S, Piacentini A, et al. Cellular and molecular events during chondrogenesis of human mesenchymal stromal cells grown in a three-dimensional hyaluronan based scaffold. Biomaterials. 2005;26(28):5677-5686.

25. Ozmen I, Naziroğlu M, Alici HA, Sahin F, Cengiz M, Eren I. Spinal morphine administration reduces the fatty acid contents in spinal cord and brain by increasing oxidative stress. Neurochem Res. 2007;32(1):19-25.

26. Cowles EA, DeRome ME, Pastizzo G, Brailey LL, Gronowicz GA. Mineralization and the expression of matrix proteins during in vivo bone development. Calcif Tissue Int. 1998;62(1):74-82.

27. Zhang J, Niu C, Ye L, et al. Identification of the haematopoietic stem cell niche and control of the niche size. Nature. 2003;425(6960): 836-841.

28. Campagnoli C, Roberts IA, Kumar S, Bennett PR, Bellantuono I, Fisk NM. Identification of mesenchymal stem/progenitor cells in human first-trimester fetal blood, liver, and bone marrow. Blood. 2001;98(8):2396-2402.

29. Yalvac ME, Rizvanov AA, Kilic E, et al. Potential role of dental stem cells in the cellular therapy of cerebral ischemia. Curr Pharm Des. 2009;15(33):3908-3916.

30. Firestone MA, Seifert S. Interaction of nonionic PEO-PPO diblock copolymers with lipid bilayers. Biomacromolecules. 2005;6(5): 2678-2687.

31. Hokett SD, Cuenin MF, O'Neal RB, et al. Pluronic polyol effects on human gingival fibroblast attachment and growth. J Periodontol. 2000;71(5):803-809.

32. Gigout A, Buschmann MD, Jolicoeur M. The fate of Pluronic F-68 in chondrocytes and CHO cells. Biotechnol Bioeng. 2008;100(5): 975-987.

33. Storlien LH, Kriketos AD, Calvert GD, Baur LA, Jenkins AB. Fatty acids, triglycerides and syndromes of insulin resistance. Prostaglandins Leukot Essent Fatty Acids. 1997;57(4-5):379-385.

34. Koga H, Engebretsen L, Brinchmann JE, Muneta T, Sekiya I. Mesenchymal stem cell-based therapy for cartilage repair: a review. Knee Surg Sports Traumatol Arthrosc. 2009;17(11):1289-1297.

35. Powers MP, Bosker H. Functional and cosmetic reconstruction of the facial lower third associated with placement of the transmandibular implant system. J Oral Maxillofac Surg. 1996;54(8): 934-942.

36. Kang X, Xie Y, Kniss DA. Adipose tissue model using three-dimensional cultivation of preadipocytes seeded onto fibrous polymer scaffolds. Tissue Eng. 2005;11(3-4):458-468.

37. Patrick CW Jr. Adipose tissue engineering: the future of breast and soft tissue reconstruction following tumor resection. Semin Surg Oncol. 2000;19(3):302-311.

38. Miller DW, Batrakova EV, Waltner TO, Alakhov VYu, Kabanov AV. Interactions of pluronic block copolymers with brain microvessel endothelial cells: evidence of two potential pathways for drug absorption. Bioconjug Chem. 1997;8(5):649-657.

39. Batrakova EV, Li S, Miller DW, Kabanov AV. Pluronic P85 increases permeability of a broad spectrum of drugs in polarized BBMEC and Caco-2 cell monolayers. Pharm Res. 1999;16(9):1366-1372.

40. Jørgensen EB, Hvidt S, Brown W, Schillén K. Effects of salts on the micellization and gelation of a triblock copolymer studied by rheology and light scattering. Macromolecules. 1997;30(8): 2355-2364.

41. Henry RL, Schmolka IR. Burn wound coverings and the use of poloxamer preparations. Critical Reviews in Biocompatibility. 1989;5:207-220

42. Jung HH, Park K, Han DK. Preparation of TGF- $\beta 1$-conjugated biodegradable pluronic F127 hydrogel and its application with adipose-derived stem cells. J Control Release. 2010;147(1): 84-91. 


\section{Publish your work in this journal}

The International Journal of Nanomedicine is an international, peerreviewed journal focusing on the application of nanotechnology in diagnostics, therapeutics, and drug delivery systems throughout the biomedical field. This journal is indexed on PubMed Central, MedLine, CAS, SciSearch $\AA$, Current Contents ${ }^{\circledR} /$ Clinical Medicine,
Journal Citation Reports/Science Edition, EMBase, Scopus and the Elsevier Bibliographic databases. The manuscript management system is completely online and includes a very quick and fair peer-review system, which is all easy to use. Visit http://www.dovepress.com/ testimonials.php to read real quotes from published authors.

Submit your manuscript here: http://www.dovepress.com/international-journal-of-nanomedicine-journal 